\title{
WEIGHTED SHARING AND UNIQUENESS OF MEROMORPHIC FUNCTIONS WITH REGARD TO MULTIPLICITY
}

\author{
PULAK SAHOO AND BISWAJIT SAHA
}

\begin{abstract}
In the paper we study the uniqueness problems of meromorphic functions whose certain nonlinear differential polynomials share certain value or fixed point with finite weight and obtain two theorems which improve two recent results due to S.S. Bhoosnurmath and S.R. Kabbur [Tamkang J. Math., 44, 11-22, Spring 2013].
\end{abstract}

\section{Introduction, definitions and results}

In this paper, by meromorphic functions we will always mean meromorphic functions in the complex plane. We adopt the standard notations in the Nevanlinna theory of meromorphic functions as explained in $[5,16,19]$. Let E denote any set of positive real numbers of finite linear measure, not necessarily the same at each occurrence. For a nonconstant meromorphic function $f$, we denote by $T(r, f)$ the Nevanlinna characteristic of $f$ and by $S(r, f)$ any quantity satisfying $S(r, f)=o\{T(r, f)\}(r \rightarrow \infty, r \notin E)$. We denote by $T(r)$ the maximum of $T(r, f)$ and $T(r, g)$, by $S(r)$ any quantity satisfying $S(r)=o\{T(r)\}(r \rightarrow \infty, r \notin E)$.

Let $f$ and $g$ be two nonconstant meromorphic functions. We say that $f$ and $g$ share the value $a \mathrm{CM}$ (counting multiplicities), if $f-a$ and $g-a$ have the same zeros with the same multiplicities. Similarly, we say that $f$ and $g$ share the value $a \mathrm{IM}$, provided that $f-a$ and $g-a$ have the same zeros ignoring multiplicities. A finite value $z_{0}$ is a fixed point of $f(z)$ if $f\left(z_{0}\right)=z_{0}$. Throughout this paper, we need the following definition.

$$
\Theta(a, f)=1-\limsup _{r \rightarrow \infty} \frac{\bar{N}(r, a ; f)}{T(r, f)},
$$

where $a$ is a value in the extended complex plane.

In 1999 I. Lahiri [6] studied the uniqueness problems of meromorphic functions when two linear differential polynomials share the same 1-points. In the same paper regarding the nonlinear differential polynomials Lahiri asked the following question.

Received May 28, 2013, accepted October 21, 2013.

2010 Mathematics Subject Classification. Primary 30D35.

Key words and phrases. Uniqueness, meromorphic function, differential polynomial, weighted sharing. 
What can be said if two nonlinear differential polynomials generated by two meromorphic functions share $1 \mathrm{CM}$ ?

Afterwards research works concerning the above question have been done by many mathematicians and continuous efforts are being put in to relax the hypothesis of the results. \{cf. [1], [3], [4], [10], [11], [12], [13], [14]\}.

In 2004 W.C. Lin and H.X. Yi [11] proved the following results which corresponded to the above question.

Theorem A. Let $f$ and $g$ be two nonconstant meromorphic functions satisfying $\Theta(\infty, f)>$ $2 /(n+1), n \geq 12$ an integer. If $f^{n}(f-1) f^{\prime}$ and $g^{n}(g-1) g^{\prime}$ share the value $1 C M$, then $f \equiv g$.

Theorem B. Let $f$ and $g$ be two nonconstant meromorphic functions, $n \geq 13$ an integer. If $f^{n}(f-1)^{2} f^{\prime}$ and $g^{n}(g-1)^{2} g^{\prime}$ share the value $1 C M$, then $f \equiv g$.

In the same year W.C. Lin and H.X. Yi [12] considered the fixed point sharing and extended Theorems A and B as follows.

Theorem C. Let $f$ and $g$ be two transcendental meromorphic functions, $n \geq 12$ an integer. If $f^{n}(f-1) f^{\prime}$ and $g^{n}(g-1) g^{\prime}$ share $z C M$, then either $f \equiv g$ or $g=\frac{(n+2)\left(1-h^{n+1}\right)}{(n+1)\left(1-h^{n+2}\right)}$ and $f=$ $\frac{(n+2) h\left(1-h^{n+1}\right)}{(n+1)\left(1-h^{n+2}\right)}$, where $h$ is a nonconstant meromorphic function.

Theorem D. Let $f$ and $g$ be two transcendental meromorphic functions, $n \geq 13$ an integer. If $f^{n}(f-1)^{2} f^{\prime}$ and $g^{n}(g-1)^{2} g^{\prime}$ share $z C M$, then $f \equiv g$.

In 2008 X.Y. Zhang, J.F. Chen and W.C. Lin [20] studied the uniqueness problem of meromorphic functions considering some general differential polynomials and proved the following result which also extends Theorems A and B.

Theorem E. Let $f$ and $g$ be two nonconstant meromorphic functions, let $n$ and $m$ be two positive integers with $n>\max \{m+10,3 m+3\}$, and let $P(z)=a_{m} z^{m}+a_{m-1} z^{m-1}+\cdots+a_{1} z+a_{0}$, where $a_{0}(\neq 0), a_{1}, \ldots, a_{m-1}, a_{m}(\neq 0)$ are complex constants. If $f^{n} P(f) f^{\prime}$ and $g^{n} P(g) g^{\prime}$ share 1 $C M$, then either $f=t g$ for a constant $t$ such that $t^{d}=1$, where $d=(n+m+1, \ldots, n+m+1-$ $i, \ldots, n+1), a_{m-i} \neq 0$ for some $i=0,1, \ldots, m$ or $f$ and $g$ satisfy the algebraic equation $R(f, g)=0$, where

$$
\begin{aligned}
R(x, y)= & x^{n+1}\left(\frac{a_{m}}{n+m+1} x^{m}+\frac{a_{m-1}}{n+m} x^{m-1}+\cdots+\frac{a_{0}}{n+1}\right)- \\
& y^{n+1}\left(\frac{a_{m}}{n+m+1} y^{m}+\frac{a_{m-1}}{n+m} y^{m-1}+\cdots+\frac{a_{0}}{n+1}\right) .
\end{aligned}
$$

In 2009 H.Y. Xu and T.B. Cao [14] considered fixed point sharing instead of value sharing in Theorem $\mathrm{E}$ and proved the following result. 
Theorem F. Let $f$ and $g$ be two transcendental meromorphic functions, and let $n$ and $m$ be two positive integers with $n>m+10$, and let $P(z)$ be defined as in Theorem $E$. If $f^{n} P(f) f^{\prime}$ and $g^{n} P(g) g^{\prime}$ share $z C M$, then the conclusions of Theorem E hold.

In 2011 R.S. Dyavanal [3] consider the uniqueness problem of meromorphic function related to the value sharing of two nonlinear differential polynomials in which the multiplicities of zeros and poles of $f$ and $g$ are taken into account. Recently S.S. Bhoosnurmath and S.R. Kabbur [2] proved the following two theorems for value sharing as well as for fixed point sharing which improve the results of Dyavanal [3].

Theorem G. Let $f$ and $g$ be two nonconstant meromorphic functions, whose zeros and poles are of multiplicities at least $s$, where $s$ is a positive integer. Let $n, m$ be two positive integers with $(n-m-1) s \geq \max \{10,2 m+3\}$ and let $P(z)$ be defined as in Theorem E. If $f^{n} P(f) f^{\prime}$ and $g^{n} P(g) g^{\prime}$ share $1 C M$, then the conclusions of Theorem E hold.

Theorem H. Let $f$ and $g$ be two transcendental meromorphic functions, whose zeros and poles are of multiplicities at least $s$, where $s$ is a positive integer. Let $n, m$ be two positive integers with $(n-m-1) s \geq \max \{10,2 m+3\}$ and let $P(z)$ be defined as in Theorem E. If $f^{n} P(f) f^{\prime}$ and $g^{n} P(g) g^{\prime}$ share $z$ CM, then the conclusions of Theorem E hold.

Now one may ask the following question which is the motivation of the paper.

Question 1.1. Is it possible simultaneously to relax the nature of sharing the value or fixed point and reduce the lower bound of $n$ in Theorems $G$ and $H$ ?

In the paper we will affirmatively solve the above question. Relaxation of the sharing can be done by the following definition known as weighted sharing of values introduced by I. Lahiri [7] which measure how close a shared value is to being shared CM or to being shared IM.

Definition 1.1. Let $k$ be a nonnegative integer or infinity. For $a \in \mathbb{C} \cup\{\infty\}$ we denote by $E_{k}(a ; f)$ the set of all $a$-points of $f$ where an $a$-point of multiplicity $\mathrm{m}$ is counted $\mathrm{m}$ times if $m \leq k$ and $\mathrm{k}+1$ times if $m>k$. If $E_{k}(a ; f)=E_{k}(a ; g)$, we say that $f, g$ share the value $a$ with weight $k$.

The definition implies that if $f, g$ share a value $a$ with weight $k$, then $z_{0}$ is an $a$-point of $f$ with multiplicity $m(\leq k)$ if and only if it is an $a$-point of $g$ with multiplicity $m(\leq k)$ and $z_{0}$ is an a-point of $f$ with multiplicity $m(>k)$ if and only if it is an a-point of $g$ with multiplicity $n(>k)$, where $\mathrm{m}$ is not necessarily equal to $\mathrm{n}$.

We write $f, g$ share $(a, k)$ to mean that $f, g$ share the value $a$ with weight k. Clearly if $f$, $g$ share $(a, k)$ then $f, g$ share $(a, p)$ for any integer $p, 0 \leq p<k$. Also we note that $f, g$ share a value $a$ IM or CM if and only if $f$, $g$ share $(a, 0)$ or $(a, \infty)$ respectively. 
We now state the main results of the paper.

Theorem 1.1. Let $f$ and $g$ be two nonconstant meromorphic functions, whose zeros and poles are of multiplicities at least $s$, where $s$ is a positive integer. Suppose that $n, m$ be two positive integers and $P(z)$ be defined as in Theorem E. Let $f^{n} P(f) f^{\prime}$ and $g^{n} P(g) g^{\prime}$ share $(1, l)$, where $l(\geq 0)$ is an integer. Then the conclusions of Theorem E hold in each of the following cases.

(i) $l \geq 2$ and $n>\max \{m+1+2 m / s, m+1+9 / s\}$;

(ii) $l=1$ and $n>\max \{m+1+2 m / s, 3 m / 2+3 / 2+21 / 2 s\}$;

(iii) $l=0$ and $n>\max \{m+1+2 m / s, 4 m+4+18 / s\}$.

Remark 1.1. Theorem 1.1 is a two fold improvement of Theorem G.

Theorem 1.2. Let $f$ and $g$ be two transcendental meromorphic functions, whose zeros and poles are of multiplicities at least $s$, where $s$ is a positive integer. Suppose that $n, m$ be two positive integers and $P(z)$ be defined as in Theorem $E$. Let $f^{n} P(f) f^{\prime}$ and $g^{n} P(g) g^{\prime}$ share $(z, l)$, where $l(\geq 0)$ is an integer. Then the conclusions of Theorem E hold in each of the following cases.

(i) $l \geq 2$ and $n>\max \{m+1+2 m / s, m+1+9 / s\}$;

(ii) $l=1$ and $n>\max \{m+1+2 m / s, 3 m / 2+3 / 2+21 / 2 s\}$;

(iii) $l=0$ and $n>\max \{m+1+2 m / s, 4 m+4+18 / s\}$.

Remark 1.2. Theorem 1.2 is a two fold improvement of Theorem H.

Though the standard definitions and notations of the value distribution theory are available in [5], we explain some definitions and notations which are used in the paper.

Definition 1.2. [7] Let $a \in \mathbb{C} \cup\{\infty\}$. We denote by $N(r, a ; f \mid=1)$ the counting function of simple $a$ points of $f$. For a positive integer $p$ we denote by $N(r, a ; f \mid \leq p)$ the counting function of those $a$-points of $f$ (counted with proper multiplicities) whose multiplicities are not greater than $p$. By $\bar{N}(r, a ; f \mid \leq p)$ we denote the corresponding reduced counting function.

Analogously we can define $N(r, a ; f \mid \geq p)$ and $\bar{N}(r, a ; f \mid \geq p)$.

Definition 1.3. [8] Let $k$ be a positive integer or infinity. We denote by $N_{k}(r, a ; f)$ the counting function of $a$-points of $f$, where an $a$-point of multiplicity $m$ is counted $m$ times if $m \leq k$ and $k$ times if $m>k$. Then

$$
N_{k}(r, a ; f)=\bar{N}(r, a ; f)+\bar{N}(r, a ; f \mid \geq 2)+\cdots+\bar{N}(r, a ; f \mid \geq k) .
$$

Clearly $N_{1}(r, a ; f)=\bar{N}(r, a ; f)$. 


\section{Lemmas}

Let $f$ and $g$ be two nonconstant meromorphic functions defined in the open complex plane $\mathbb{C}$. We denote by $H$ the function as follows:

$$
H=\left(\frac{f^{\prime \prime}}{f^{\prime}}-\frac{2 f^{\prime}}{f-1}\right)-\left(\frac{g^{\prime \prime}}{g^{\prime}}-\frac{2 g^{\prime}}{g-1}\right) .
$$

Lemma 2.1 ([15]). Let $f$ be a transcendental meromorphic function, and let $P_{n}(f)$ be a polynomial in $f$ of the form

$$
P_{n}(f)=a_{n} f^{n}(z)+a_{n-1} f^{n-1}(z)+\cdots+a_{1} f(z)+a_{0},
$$

where $a_{n}(\neq 0), a_{n-1}, \ldots, a_{1}, a_{0}$ are complex numbers. Then

$$
T\left(r, P_{n}(f)\right)=n T(r, f)+O(1) .
$$

Lemma 2.2 ([17]). Let $f$ be a nonconstant meromorphic function. Then

$$
N\left(r, 0 ; f^{(k)}\right) \leq N(r, 0 ; f)+k \bar{N}(r, \infty ; f)+S(r, f) .
$$

Lemma 2.3 ([7]). Let $f$ and $g$ be two nonconstant meromorphic functions sharing $(1,2)$. Then one of the following three cases hold:

(i) $T(r) \leq N_{2}(r, 0 ; f)+N_{2}(r, 0 ; g)+N_{2}(r, \infty ; f)+N_{2}(r, \infty ; g)+S(r)$,

(ii) $f=g$,

(iii) $f g=1$.

Lemma 2.4 ([1]). Let $f$ and $g$ be two nonconstant meromorphic functions that share $(1, m)$ and $H \not \equiv 0$. Then

(i) if $m=1$ then $T(r, f) \leq N_{2}(r, 0 ; f)+N_{2}(r, 0 ; g)+N_{2}(r, \infty ; f)+N_{2}(r, \infty ; g)+\frac{1}{2} \bar{N}(r, 0 ; f)+$ $\frac{1}{2} \bar{N}(r, \infty ; f)+S(r, f)+S(r, g)$

(ii) if $m=0$ then $T(r, f) \leq N_{2}(r, 0 ; f)+N_{2}(r, 0 ; g)+N_{2}(r, \infty ; f)+N_{2}(r, \infty ; g)+2 \bar{N}(r, 0 ; f)+$ $\bar{N}(r, 0 ; g)+2 \bar{N}(r, \infty ; f)+\bar{N}(r, \infty ; g)+S(r, f)+S(r, g)$.

Lemma 2.5 ([18]). Let $f$ and $g$ be two nonconstant meromorphic functions and $H=0$. If

$$
\limsup _{r \rightarrow \infty, r \notin E} \frac{\bar{N}(r, 0 ; f)+\bar{N}(r, 0 ; g)+\bar{N}(r, \infty ; f)+\bar{N}(r, \infty ; g)}{T(r)}<1
$$

then $f=g$ or $f g=1$, where $E$ is a set of finite linear measure and not necessarily the same at each of its occurance. 
Lemma 2.6. Let $f$ and $g$ be two transcendental meromorphic functions, whose zeros and poles are of multiplicities at least $s$, where $s$ is a positive integer. Let $P(z)$ be defined as in Theorem $E$, and let $n$ and $m$ be two positive integers such that $(n+m-1) p>2 m(1+1 / s)$ where $p$ is the number of distinct roots of $P(z)=0$. Then

$$
f^{n} P(f) f^{\prime} g^{n} P(g) g^{\prime} \not \equiv z^{2} .
$$

Proof. If possible, we assume that

$$
f^{n} P(f) f^{\prime} g^{n} P(g) g^{\prime}=z^{2} .
$$

Let

$$
P(z)=a_{m}\left(z-d_{1}\right)^{l_{1}}\left(z-d_{2}\right)^{l_{2}} \cdots\left(z-d_{i}\right)^{l_{i}} \cdots\left(z-d_{p}\right)^{l_{p}},
$$

where $\sum_{i=1}^{p} l_{i}=m, 1 \leq p \leq m ; d_{i} \neq d_{j}, i \neq j, 1 \leq i, j \leq p$; $d_{i}$ 's are nonzero constants and $l_{i}$ 's are positive integers, $i=1,2, \ldots, p$. Let $z_{0}(\neq 0, \infty)$ be a zero of $f$ with multiplicity $p_{0}(\geq s)$. Then $z_{0}$ is a pole of $g$ with multiplicity $q_{0}(\geq s)$, say. From (2.1) we get

$$
n p_{0}+p_{0}-1=(n+m+1) q_{0}+1
$$

and so

$$
m q_{0}+2=(n+1)\left(p_{0}-q_{0}\right)
$$

From (2.2) we get $q_{0} \geq \frac{n-1}{m}$ and so

$$
p_{0} \geq \frac{1}{n+1}\left[\frac{(n+m+1)(n-1)}{m}+2\right]=\frac{n+m-1}{m} .
$$

Let $z_{1}(\neq 0, \infty)$ be a zero of $P(f)$ of order $p_{1}$ and be a zero of $f-d_{i}$ of order $q_{i}$ for $i=1,2, \ldots, p$. Then $p_{1}=l_{i} q_{i}$ for $i=1,2, \ldots, p$. Then $z_{1}$ is a pole of $g$ with multiplicity $q(\geq s)$, say. So from (2.1) we get

$$
\begin{aligned}
q_{i} l_{i}+q_{i}-1 & =(n+m+1) q+1 \\
& \geq(n+m+1) s+1
\end{aligned}
$$

i.e.,

$$
q_{i} \geq \frac{(n+m+1) s+2}{l_{i}+1}
$$

for $i=1,2, \ldots, p$.

Suppose that $z_{2}(\neq 0, \infty)$ be a pole of $f$. Then from $(2.1), z_{2}$ is either a zero of $g^{n} P(g)$ or a zero of $g^{\prime}$. Therefore

$$
\bar{N}(r, \infty ; f) \leq \bar{N}(r, 0 ; g)+\sum_{i=1}^{p} \bar{N}\left(r, d_{i} ; g\right)+\bar{N}_{0}\left(r, 0 ; g^{\prime}\right)+S(r, f)+S(r, g)
$$




$$
\begin{aligned}
\leq & \left(\frac{m}{n+m-1}+\frac{m+p}{(n+m+1) s+2}\right) T(r, g)+\bar{N}_{0}\left(r, 0 ; g^{\prime}\right) \\
& +S(r, f)+S(r, g),
\end{aligned}
$$

where $\bar{N}_{0}\left(r, 0 ; g^{\prime}\right)$ denotes the reduced counting function of those zeros of $g^{\prime}$ which are not the zeros of $g P(g)$. Then by the second fundamental theorem of Nevanlinna we get

$$
\begin{aligned}
p T(r, f) \leq & \bar{N}(r, \infty ; f)+\bar{N}(r, 0 ; f)+\sum_{i=1}^{p} \bar{N}\left(r, d_{i} ; f\right)-\bar{N}_{0}\left(r, 0 ; f^{\prime}\right) \\
& +S(r, f) \\
\leq & \left(\frac{m}{n+m-1}+\frac{m+p}{(n+m+1) s+2}\right)\{T(r, f)+T(r, g)\} \\
& +\bar{N}_{0}\left(r, 0 ; g^{\prime}\right)-\bar{N}_{0}\left(r, 0 ; f^{\prime}\right)+S(r, f)+S(r, g) .
\end{aligned}
$$

Similarly

$$
\begin{aligned}
p T(r, g) \leq & \left(\frac{m}{n+m-1}+\frac{m+p}{(n+m+1) s+2}\right)\{T(r, f)+T(r, g)\} \\
& +\bar{N}_{0}\left(r, 0 ; f^{\prime}\right)-\bar{N}_{0}\left(r, 0 ; g^{\prime}\right)+S(r, f)+S(r, g) .
\end{aligned}
$$

Adding (2.3) and (2.4) we obtain

$$
\left(p-\frac{2 m}{n+m-1}-\frac{2(m+p)}{(n+m+1) s+2}\right)\{T(r, f)+T(r, g)\} \leq S(r, f)+S(r, g),
$$

contradicting with the fact that $(n+m-1) p>2 m(1+1 / s)$. This proves the lemma.

Note 2.1. If $P(z)=0$ has only one root of multiplicity $m$ then the above lemma holds for $n>$ $m+1+2 m / s$. If all the roots of $P(z)=0$ are distinct then the lemma holds for $n>3-m+2 / s$.

Proceeding similarly as in Lemma 2.6 we obtain the following lemma.

Lemma 2.7. Let $f$ and $g$ be two nonconstant meromorphic functions, whose zeros and poles are of multiplicities at least $s$, where $s$ is a positive integer. Let $P(z)$ be defined as in Theorem $E$, and let $n$ and $m$ be two positive integers such that $(n+m-1) p>2 m(1+1 / s)$ where $p$ is the number of distinct roots of $P(z)=0$. Then

$$
f^{n} P(f) f^{\prime} g^{n} P(g) g^{\prime} \not \equiv 1 \text {. }
$$

Lemma 2.8. Let $f$ and $g$ be two transcendental meromorphic functions and

$$
F=f^{n+1}\left[\frac{a_{m}}{n+m+1} f^{m}+\frac{a_{m-1}}{n+m} f^{m-1}+\cdots+\frac{a_{0}}{n+1}\right]
$$

and

$$
G=g^{n+1}\left[\frac{a_{m}}{n+m+1} g^{m}+\frac{a_{m-1}}{n+m} g^{m-1}+\cdots+\frac{a_{0}}{n+1}\right],
$$

where $a_{0}(\neq 0), a_{1}, \ldots, a_{m-1}, a_{m}(\neq 0)$ are complex constants. Further let $F_{0}=\frac{F^{\prime}}{z}$ and $G_{0}=\frac{G^{\prime}}{z}$. Then $S\left(r, F_{0}\right)$ and $S\left(r, G_{0}\right)$ are replaceable by $S(r, f)$ and $S(r, g)$ respectively. 
Proof. Since $f$ and $g$ be two transcendental meromorphic functions we have

$$
T(r, z)=o\{T(r, f)\} \text { and } T(r, z)=o\{T(r, g)\} .
$$

Therefore using Lemma 2.1 we obtain

$$
\begin{aligned}
T\left(r, F_{0}\right) & \leq T\left(r, F^{\prime}\right)+T(r, z) \\
& \leq 2 T(r, F)+S(r, f) \\
& =2(n+m+1) T(r, f)+S(r, f)
\end{aligned}
$$

and similarly

$$
T\left(r, G_{0}\right) \leq 2(n+m+1) T(r, g)+S(r, g) .
$$

This proves the lemma.

Lemma 2.9. Let $F, G, F_{0}$ and $G_{0}$ be defined as in Lemma 2.8. We define $F=f^{n+1} F^{*}$ and $G=g^{n+1} G^{*}$ where

$$
F^{*}=\left[\frac{a_{m}}{n+m+1} f^{m}+\frac{a_{m-1}}{n+m} f^{m-1}+\cdots+\frac{a_{0}}{n+1}\right]
$$

and

$$
G^{*}=\left[\frac{a_{m}}{n+m+1} g^{m}+\frac{a_{m-1}}{n+m} g^{m-1}+\cdots+\frac{a_{0}}{n+1}\right] .
$$

Then

(i) $T(r, F) \leq T\left(r, F_{0}\right)+N(r, 0 ; f)+\sum_{i=1}^{m} N\left(r, c_{i} ; f\right)-\sum_{j=1}^{m} N\left(r, d_{j} ; f\right)-N\left(r, 0 ; f^{\prime}\right)+S(r, f)$,
(ii) $T(r, G) \leq T\left(r, G_{0}\right)+N(r, 0 ; g)+\sum_{i=1}^{m} N\left(r, c_{i} ; g\right)-\sum_{j=1}^{m} N\left(r, d_{j} ; g\right)-N\left(r, 0 ; g^{\prime}\right)+S(r, g)$, where $c_{1}, c_{2}, \ldots, c_{m}$ are the roots of the equation

$$
\frac{a_{m}}{n+m+1} z^{m}+\frac{a_{m-1}}{n+m} z^{m-1}+\cdots+\frac{a_{0}}{n+1}=0,
$$

and $d_{1}, d_{2}, \ldots, d_{m}$ are the roots of the equation $P(z)=0$.

Proof. Noting that $f$ and $g$ be two transcendental meromorphic functions the proof of the lemma can be carried out in the line of the proof of Lemma 11 [13].

Following lemma can be proved in the line of Lemma 2.10 [9].

Lemma 2.10. Let $F$ and $G$ be defined as in Lemma 2.8, where $m$ and $n(>m+2)$ are positive integers. Then $F^{\prime}=G^{\prime}$ implies $F=G$. 


\section{Proof of the Theorem}

Proof of Theorem 1.2. Let $F, G, F_{0}$ and $G_{0}$ be defined as in Lemma 2.8. Then $F_{0}$ and $G_{0}$ share $(1, l)$ where $l(\geq 0)$ is an integer. Now we discuss following three cases separately.

Case 3.1. Let $l \geq 2$. Then one of the possibilities of Lemma 2.3 hold. If possible, suppose that

$$
\begin{aligned}
T_{0}(r) \leq & N_{2}\left(r, 0 ; F_{0}\right)+N_{2}\left(r, 0 ; G_{0}\right)+N_{2}\left(r, \infty ; F_{0}\right) \\
& +N_{2}\left(r, \infty ; G_{0}\right)+S\left(r, F_{0}\right)+S\left(r, G_{0}\right),
\end{aligned}
$$

where $T_{0}(r)=\max \left\{T\left(r, F_{0}\right), T\left(r, G_{0}\right)\right\}$. Using Lemmas 2.2, 2.8, 2.9 and (3.1) we obtain

$$
\begin{aligned}
T(r, F) \leq & T\left(r, F_{0}\right)+N(r, 0 ; f)+\sum_{i=1}^{m} N\left(r, c_{i} ; f\right)-\sum_{j=1}^{m} N\left(r, d_{j} ; f\right) \\
& -N\left(r, 0 ; f^{\prime}\right)+S(r, f) \\
\leq & N_{2}\left(r, 0 ; F_{0}\right)+N_{2}\left(r, 0 ; G_{0}\right)+N_{2}\left(r, \infty ; F_{0}\right)+N_{2}\left(r, \infty ; G_{0}\right) \\
& +N(r, 0 ; f)+\sum_{i=1}^{m} N\left(r, c_{i} ; f\right)-\sum_{j=1}^{m} N\left(r, d_{j} ; f\right) \\
& -N\left(r, 0 ; f^{\prime}\right)+S(r, f)+S(r, g) \\
\leq & 2 \bar{N}(r, 0 ; f)+\sum_{j=1}^{m} N\left(r, d_{j} ; f\right)+N\left(r, 0 ; f^{\prime}\right)+2 \bar{N}(r, \infty ; f) \\
& +2 \bar{N}(r, 0 ; g)+\sum_{j=1}^{m} N\left(r, d_{j} ; g\right)+N\left(r, 0 ; g^{\prime}\right)+2 \bar{N}(r, \infty ; g) \\
& +N(r, 0 ; f)+\sum_{i=1}^{m} N\left(r, c_{i} ; f\right)-\sum_{j=1}^{m} N\left(r, d_{j} ; f\right) \\
& -N\left(r, 0 ; f^{\prime}\right)+S(r, f)+S(r, g) \\
= & 2 \bar{N}(r, 0 ; f)+N(r, 0 ; f)+\sum_{i=1}^{m} N\left(r, c_{i} ; f\right)+2 \bar{N}(r, \infty ; f) \\
& 2 \bar{N}(r, 0 ; g)+N\left(r, 0 ; g^{\prime}\right)+\sum_{j=1}^{m} N\left(r, d_{j} ; g\right)++2 \bar{N}(r, \infty ; g) \\
& +S(r, f)+S(r, g) \\
\leq & (m+1+4 / s) T(r, f)+(m+1+5 / s) T(r, g)+S(r, f)+S(r, g) . \\
&
\end{aligned}
$$

Therefore by Lemma 2.1 we obtain

$$
(n+m+1) T(r, f) \leq(2 m+2+9 / s) T(r)+S(r) .
$$

Similarly

$$
(n+m+1) T(r, g) \leq(2 m+2+9 / s) T(r)+S(r) \text {. }
$$


Combining the above two inequalities we get

$$
(n-m-1-9 / s) T(r) \leq S(r),
$$

which contradicts the fact that $n>\max \{m+1+2 m / s, m+1+9 / s\}$. Therefore by Lemma 2.3 we have either $F_{0} G_{0}=1$ or $F_{0}=G_{0}$. Since $F_{0} G_{0} \not \equiv 1$, by Lemma 2.6, using Lemma 2.10 we obtain $F=G$. That is

$$
\begin{array}{r}
f^{n+1}\left[\frac{a_{m}}{n+m+1} f^{m}+\frac{a_{m-1}}{n+m} f^{m-1}+\cdots+\frac{a_{0}}{n+1}\right]=g^{n+1} \\
{\left[\frac{a_{m}}{n+m+1} g^{m}+\frac{a_{m-1}}{n+m} g^{m-1}+\cdots+\frac{a_{0}}{n+1}\right] .}
\end{array}
$$

Let $h=\frac{f}{g}$. If $h$ is a constant, replacing $f=g h$ in (3.2) we get

$$
\begin{array}{r}
\frac{a_{m}}{m+n+1} g^{m}\left(h^{n+m+1}-1\right)+\frac{a_{m-1}}{m+n} g^{m-1}\left(h^{n+m}-1\right)+\cdots \\
+\frac{a_{0}}{n+1}\left(h^{n+1}-1\right)=0,
\end{array}
$$

which implies $h^{d}=1$, where $d=(n+m+1, \ldots, n+m+1-i, \ldots, n+1), a_{m-i} \neq 0$ for some $i=$ $0,1, \ldots, m$. Thus $f=$ tg for a constant $t$ such that $t^{d}=1, d=(n+m+1, \ldots, n+m+1-i, \ldots, n+1)$, $a_{m-i} \neq 0$ for some $i=0,1, \ldots, m$.

If $h$ is not a constant, then from (3.2) we can say that $f$ and $g$ satisfy the algebraic equation $R(f, g)=0$, where

$$
\begin{aligned}
R(x, y)= & x^{n+1}\left(\frac{a_{m}}{n+m+1} x^{m}+\frac{a_{m-1}}{n+m} x^{m-1}+\cdots+\frac{a_{0}}{n+1}\right)- \\
& y^{n+1}\left(\frac{a_{m}}{n+m+1} y^{m}+\frac{a_{m-1}}{n+m} y^{m-1}+\cdots+\frac{a_{0}}{n+1}\right) .
\end{aligned}
$$

This completes the proof of Case 3.1.

Case 3.2. We suppose that $l=1$ and $H \not \equiv 0$. Then using Lemmas 2.2, 2.4(i), 2.8 and 2.9 we obtain

$$
\begin{aligned}
T(r, F) \leq & T\left(r, F_{0}\right)+N(r, 0 ; f)+\sum_{i=1}^{m} N\left(r, c_{i} ; f\right)-\sum_{j=1}^{m} N\left(r, d_{j} ; f\right) \\
& -N\left(r, 0 ; f^{\prime}\right)+S(r, f) \\
\leq & N_{2}\left(r, 0 ; F_{0}\right)+N_{2}\left(r, 0 ; G_{0}\right)+N_{2}\left(r, \infty ; F_{0}\right)+N_{2}\left(r, \infty ; G_{0}\right) \\
& +\frac{1}{2} \bar{N}\left(r, 0 ; F_{0}\right)+\frac{1}{2} \bar{N}\left(r, \infty ; F_{0}\right)+N(r, 0 ; f)+\sum_{i=1}^{m} N\left(r, c_{i} ; f\right) \\
& -\sum_{j=1}^{m} N\left(r, d_{j} ; f\right)-N\left(r, 0 ; f^{\prime}\right)+S(r, f)+S(r, g)
\end{aligned}
$$




$$
\begin{aligned}
\leq & 2 \bar{N}(r, 0 ; f)+\sum_{j=1}^{m} N\left(r, d_{j} ; f\right)+N\left(r, 0 ; f^{\prime}\right)+2 \bar{N}(r, \infty ; f) \\
& +2 \bar{N}(r, 0 ; g)+\sum_{j=1}^{m} N\left(r, d_{j} ; g\right)+N\left(r, 0 ; g^{\prime}\right)+2 \bar{N}(r, \infty ; g) \\
& +\frac{1}{2} \bar{N}(r, 0 ; f)+\frac{1}{2} \sum_{j=1}^{m} N\left(r, d_{j} ; f\right)+\frac{1}{2} \bar{N}\left(r, 0 ; f^{\prime}\right)+\frac{1}{2} \bar{N}(r, \infty ; f) \\
& +N(r, 0 ; f)+\sum_{i=1}^{m} N\left(r, c_{i} ; f\right)-\sum_{j=1}^{m} N\left(r, d_{j} ; f\right)-N\left(r, 0 ; f^{\prime}\right) \\
& +S(r, f)+S(r, g) \\
= & \frac{5}{2} \bar{N}(r, 0 ; f)+\frac{1}{2} \sum_{j=1}^{m} N\left(r, d_{j} ; f\right)+N(r, 0 ; f)+\frac{1}{2} \bar{N}\left(r, 0 ; f^{\prime}\right)+\frac{5}{2} \bar{N}(r, \infty ; f) \\
& +\sum_{i=1}^{m} N\left(r, c_{i} ; f\right)+2 \bar{N}(r, 0 ; g)+\sum_{j=1}^{m} N\left(r, d_{j} ; g\right)+N\left(r, 0 ; g^{\prime}\right)+2 \bar{N}(r, \infty ; g) \\
& +S(r, f)+S(r, g) \\
\leq & (3 m / 2+3 / 2+11 / 2 s) T(r, f)+(m+1+5 / s) T(r, g)+S(r, f)+S(r, g) .
\end{aligned}
$$

So by Lemma 2.1 we get

$$
(n+m+1) T(r, f) \leq(5 m / 2+5 / 2+21 / 2 s) T(r)+S(r) .
$$

Similarly

$$
(n+m+1) T(r, g) \leq(5 m / 2+5 / 2+21 / 2 s) T(r)+S(r) .
$$

Combining the above two inequalities we obtain

$$
(n-3 m / 2-3 / 2-21 / 2 s) T(r) \leq S(r),
$$

a contradiction with the fact that $n>\max \{m+1+2 m / s, 3 m / 2+3 / 2+21 / 2 s\}$. Therefore $H=0$. Since $f$ and $g$ are transcendental meromorphic functions, by Lemma 2.1 we get

$$
\begin{aligned}
(n+m) T(r, f) & =T\left(r, f^{n} P(f)\right)+S(r, f) \\
& \leq T\left(r, F^{\prime}\right)+T\left(r, f^{\prime}\right)+S(r, f) \\
& \leq T\left(r, F_{0}\right)+2 T(r, f)+S(r, f)
\end{aligned}
$$

and so

$$
T\left(r, F_{0}\right) \geq(n+m-2) T(r, f)+S(r, f) .
$$

Similarly

$$
T\left(r, G_{0}\right) \geq(n+m-2) T(r, g)+S(r, g) .
$$


Also we see by Lemma 2.2 that

$$
\begin{aligned}
& \bar{N}\left(r, 0 ; F_{0}\right)+\bar{N}\left(r, \infty ; F_{0}\right)+\bar{N}\left(r, 0 ; G_{0}\right)+\bar{N}\left(r, \infty ; G_{0}\right) \\
\leq & \bar{N}(r, 0 ; f)+\sum_{j=1}^{m} N\left(r, d_{j} ; f\right)+\bar{N}\left(r, 0 ; f^{\prime}\right)+\bar{N}(r, \infty ; f) \\
& +\bar{N}(r, 0 ; g)+\sum_{j=1}^{m} N\left(r, d_{j} ; g\right)+\bar{N}\left(r, 0 ; g^{\prime}\right)+\bar{N}(r, \infty ; g) \\
& +S(r, f)+S(r, g) \\
\leq & (m+1+3 / s) T(r, f)+(m+1+3 / s) T(r, g)+S(r, f)+S(r, g) \\
\leq & \frac{2 m+2+6 / s}{n+m-2} T_{0}(r)+S(r),
\end{aligned}
$$

where $S_{0}(r)=o\left\{T_{0}(r)\right\}$ as $r \rightarrow \infty$ possibly outside a set of finite linear measure. Since $n>$ $\max \{m+1+2 m / s, 3 m / 2+3 / 2+21 / 2 s\}$, from above it follows that

$$
\limsup _{r \rightarrow \infty, r \notin E} \frac{\bar{N}\left(r, 0 ; F_{0}\right)+\bar{N}\left(r, \infty ; F_{0}\right)+\bar{N}\left(r, 0 ; G_{0}\right)+\bar{N}\left(r, \infty ; G_{0}\right)}{T_{0}(r)}<1,
$$

which gives by Lemma 2.5 either $F_{0} G_{0}=1$ or $F_{0}=G_{0}$. Using Lemmas 2.6, 2.10 and proceeding as in Case 3.1 we obtain the result of Case 3.2.

Case 3.3. This case can be carried out by Lemma 2.4(ii) and proceeding in the like manner as Case 3.2. Here we omit the details. This proves the theorem.

Proof of Theorem 1.1. Using Lemma 2.7 the theorem can be proved as the proof of Theorem 1.2 .

\section{References}

[1] A. Banerjee, Meromorphic functions sharing one value, Int. J. Math. Math. Sci., 22(2005), 3587-3598.

[2] S. S. Bhoosnurmath and S. R. Kabbur, Uniqueness and value sharing of meromorphic functions with regard to multiplicity, Tamkang J. Math., 44 (2013), 11-22.

[3] R. S. Dyavanal, Uniqueness and value sharing of differential polynomials of meromorphic functions, J. Math. Anal. Appl., 374 (2011), 335-345.

[4] C. Y. Fang and M. L. Fang, Uniqueness of meromorphic functions and differential polynomials, Comput. Math. Appl., 44 (2002), 607-617.

[5] W.K. Hayman, Meromorphic Functions, The Clarendon Press, Oxford (1964).

[6] I. Lahiri, Uniqueness of meromorphic functions when two linear differential polynomials share the same 1points, Ann. Polon. Math., 71 (1999), 113-128.

[7] I. Lahiri, Weighted value sharing and uniqueness of meromorphic functions, Complex Var. Theory Appl., 46 (2001), 241-253.

[8] I. Lahiri, Weighted sharing of three values, Z. Anal. Anwendungen, 23 (2004), 237-252.

[9] I. Lahiri and A. Sarkar, Nonlinear differential polynomials sharing 1-points with weight two, Chinese J. Contemp. Math., 25 (2004), 325-334. 
[10] I. Lahiri and P. Sahoo, Uniqueness of meromorphic functions when two nonlinear differential polynomials share a small function, Arch. Math. (Brno), 44 (2008), 201-210.

[11] W. C. Lin and H. X. Yi, Uniqueness theorems for meromorphic functions, Indian J. Pure Appl. Math., 35 (2004), 121-132.

[12] W. C. Lin and H. X. Yi, Uniqueness theorems for meromorphic functions concerning fixed points, Complex Variables, 49 (2004), 793-806.

[13] P. Sahoo and S. Seikh, Nonlinear differential polynomials sharing a small function, Math. Vesnik, 65 (2013), 151-165.

[14] H. Y. Xu and T. B. Cao, Uniqueness of entire or meromorphic functions sharing one value or a function with finite weight, J. Inequal. Pure Appl. Math., 10 (2009), Issue 3, Art. 88.

[15] C. C. Yang, On deficiencies of differential polynomials II, Math. Z., 125 (1972), 107-112.

[16] L. Yang, Value Distribution Theory, Springer- Verlag, Berlin, 1993.

[17] H. X. Yi, Uniqueness of meromorphic functions and a question of C. C. Yang, Complex Var. Theory Appl., 14 (1990), 169-176.

[18] H. X. Yi, Meromorphic functions that share one or two values, Complex Var. Theory Appl., 28 (1995), 1-11.

[19] H. X. Yi and C. C. Yang, Uniqueness Theory of Meromorphic Functions, Science Press, Beijing, 1995.

[20] X. Y. Zhang, J. F. Chen and W. C. Lin, Entire or meromorphic functions sharing one value, Comput. Math. Appl., 56 (2008), 1876-1883.

Department of Mathematics, University of Kalyani, West Bengal-741235, India.

E-mail: sahoopulak@yahoo.com; sahoopulak1@gmail.com

Department of Mathematics, Ghurni High School(H.S), Krishnagar, West Bengal, 741101, India.

E-mail: sahaanjan11@gmail.com 